\title{
Are We Allowed to Limit Surgical Aggressiveness With Small Rectal Gastrointestinal Stromal Tumors?
}

\author{
Marco Fiore, $\mathrm{MD}^{1}$ (D), Chandrajit P. Raut, $\mathrm{MD}, \mathrm{MSc}^{2}$, and Alessandro Gronchi, $\mathrm{MD}^{\mathbf{1}}$ \\ ${ }^{1}$ Department of Surgery, Fondazione IRCCS Istituto Nazionale dei Tumori, Milan, Italy; ${ }^{2}$ Department of Surgery, Brigham \\ and Women's Hospital Center for Sarcoma and Bone Oncology, Dana-Farber Cancer Institute Harvard Medical School, \\ Boston, MA
}

This Annals of Surgical Oncology issue includes a retrospective study evaluating the role of neodjuvant therapy and different surgical approaches in the management of anorectal gastrointestinal stromal tumors (GISTs). ${ }^{1}$ This is the largest series of anorectal GISTs reported to date, obtained from the National Cancer Data Base (NCDB), which includes more than 1500 centers in the United States.

Concerning the surgical approach, Hawkins et al. ${ }^{1}$ distinguished between anorectal GISTs treated with radical excision and those treated with local resection, concluding that local resection is acceptable, with no difference in survival compared with radical excision for tumors smaller than $5 \mathrm{~cm}$. Indeed, the equipoise between conservative and extended surgical resection of GISTs (any site) has been debated because an effective cytoreductive therapy by tyrosin-kinase inhibitors (TKIs) became available during the last 15 years. $^{2}$

To date, reported data on rectal GISTs have been limited to small retrospective series of patients undergoing surgery at referral centers and to the few patients included in the adjuvant prospective randomized studies (10 patients in the ACOSOG Z9001 study, 35 patients in the SSG/AIO study, and an unknown number of patients in the EORTC-STSBG study). ${ }^{3-5}$

However, due to the nature of the NCDB, the analysis of Hawkins et al. ${ }^{1}$ has several shortcomings. First, only overall survival (OS) is available. No data on local control after local resection or radical excision are retrievable. If

\footnotetext{
(C) Society of Surgical Oncology 2016

First Received: 21 October 2016; Published Online: 29 November 2016

M. Fiore, MD

e-mail: marco.fiore@istitutotumori.mi.it
}

the two approaches result in different local control rates, which in the long run may translate into different OS, these data are unavailable in the NCDB. In the era of TKIs, which significantly prolong OS upon recurrence, mediumterm OS may not reflect the real impact on the disease control of the initial strategy. Therefore, the analysis of Hawkins et al. ${ }^{1}$ on the role of local resection versus radical excision should be cautiously interpreted concerning the impact of surgical technique on local recurrence.

Second, tumor size is available only at the time of surgery, and the initial tumor size for those patients who received preoperative medical therapy is not available. Although the possible benefit of adjuvant imatinib mesylate for larger tumors is intuitive, a clean analysis to find a possible risk cutoff cannot be performed.

Finally, the genotype of GISTs was unknown. The mutational status of Kit and PDGFRA (a pathogenetic mutation in one of these two genes is found in more than $85 \%$ of GISTs) carries prognostic implications and is a useful predictive tool for predicting sensitivity to TKIs. Therefore, it is important to obtain this information before recommending any treatment strategy, especially for patients with tumors occurring at this challenging site.

Despite these limitations, the study by Hawkins et al. ${ }^{1}$ still provides important additional knowledge to the community. In general, patients affected by rare disease such as rectal GISTs should be treated at referral centers by a team of experienced clinicians, essentially due to the multidisciplinary approach this disease may often require. However, the indications for local resection versus radical excision for rectal GIST in the series of Hawkins et al. ${ }^{1}$ very likely reflect the approach commonly used for rectal cancer. In fact, the larger number of centers contributing to NCDB covers a wide community hospital network in the United States and more than sarcoma centers alone. 

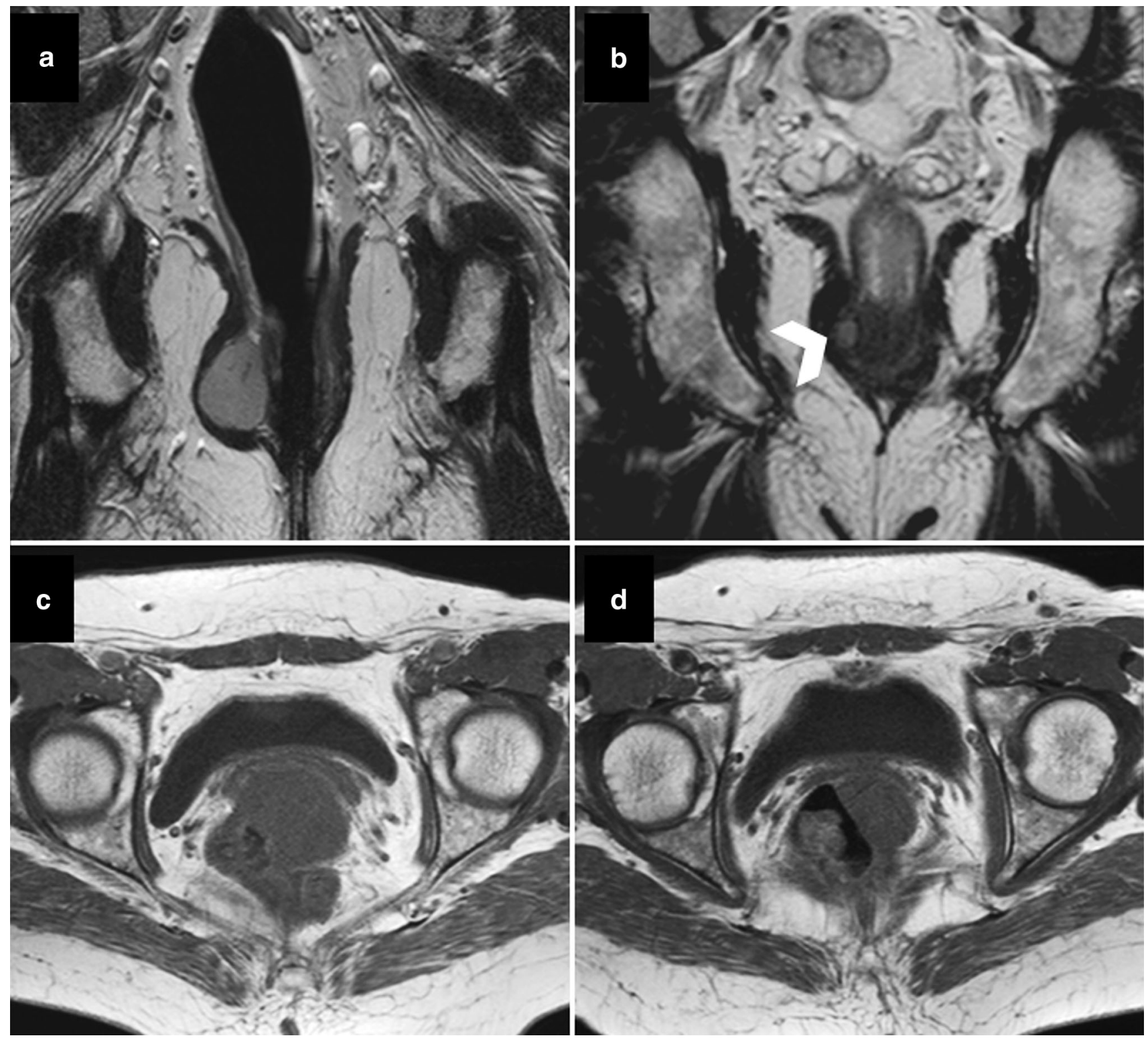

FIG. 1 Anorectal gastrointestinal stromal tumors (GISTs) with an exophytic pattern of growth. a KIT Exon 11 mutant rectal GIST in a 61-year-old man arising from the anorectal region and displacing the levator ani muscle [baseline $\mathrm{T} 1 \mathrm{w}$ magnetic resonance imaging (MRI)]. b Partial response at the preoperative reevaluation after 6 months of imatinib mesylate $400 \mathrm{mg}$ per day. c KIT Exon 11

As detailed by Hawkins et al., ${ }^{1}$ local resection includes transanal excision, transanal endoscopic microsurgery (TEMS), transanal minimally invasive surgery (TAMIS), and any other local tumor excision. Nonetheless, GISTs are biologically different from epithelial tumors such as rectal cancer. For this reason, the correlation between anatomic local tumor extent and surgical procedure may differ from that for rectal cancer.

Another option for a technical approach is a transperineal approach. ${ }^{6}$ As a matter of fact, the presentation of

mutant rectal GIST in a 53-year-old woman growing in the rectovaginal space (baseline T1w MRI). d Partial response after 10 months of imatinib mesylate $400 \mathrm{mg}$ per day

anorectal GISTs as exophytic nodules immediately proximal to the levator muscles is not uncommon (Fig. 1). In this setting, a transperineal local resection may be considered as an alternative option to circumferential resection techniques (Fig. 2), to obtain clearance of surrounding tissues and to limit the extent of rectal wall resection.

Indeed, it is very important to minimize the risk of positive margins, at both the rectal and perirectal sites, because this is associated with a significantly increased risk of local recurrence and worse survival. ${ }^{7}$ It currently is well 

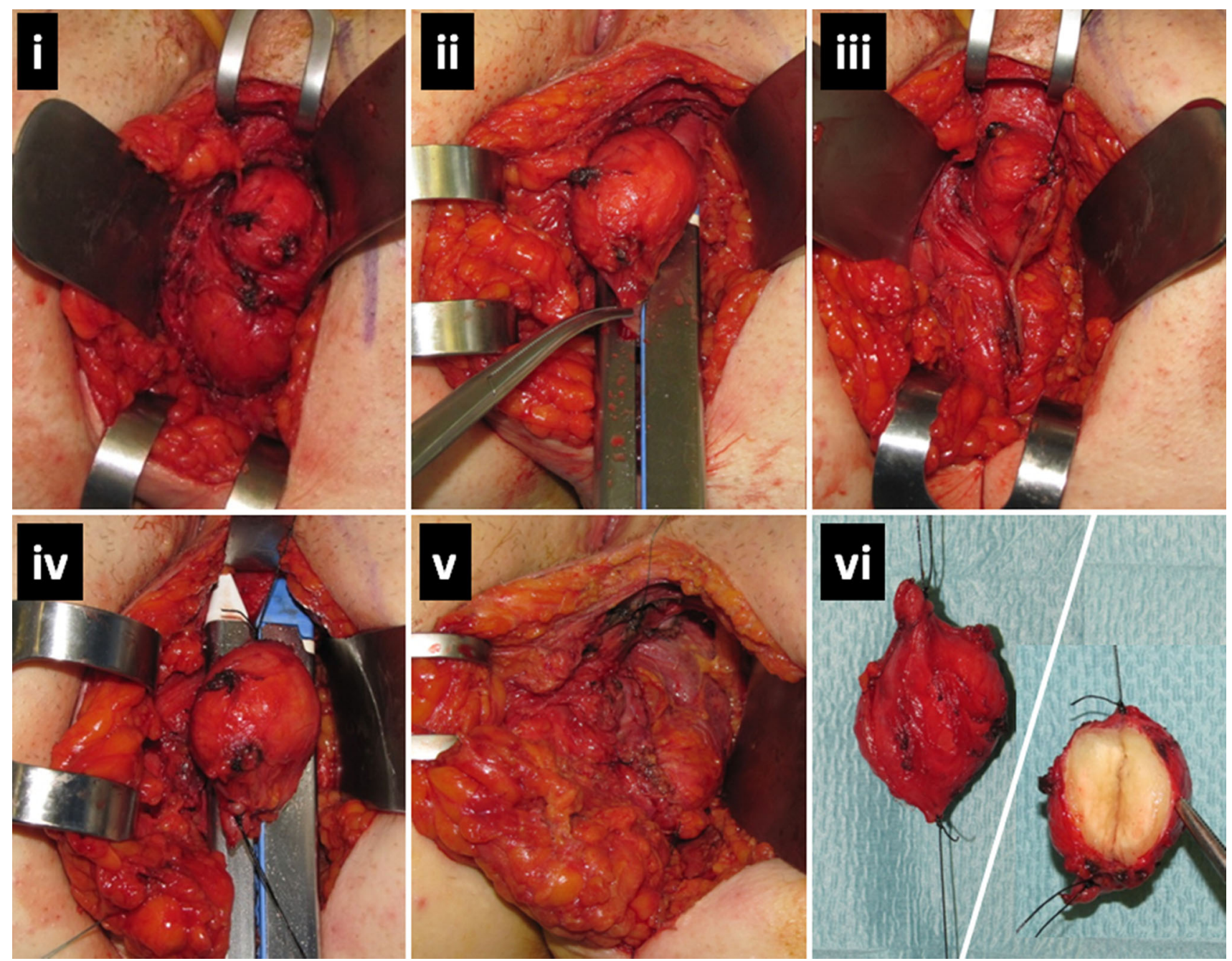

FIG. 2 Local excision of rectal GIST after imatinib therapy (same patient as in Fig. 1, panels c, d). $i$ Exposure of residual mass through a transperineal left pararectal incision. $i i-i v$ Atypical full-thickness

known how TKI may be used as preoperative therapy for rectal GISTs to downsize the tumor and limit the extent of surgery as much as possible without compromising the quality of surgical margins. ${ }^{7}$ A local excision then may be an option when the tumor has been sufficiently downsized. Which local approach is better has not been studied to date and, given the rarity of the disease, likely never will be studied. The study of Hawkins et al. ${ }^{1}$ does not provide any definitive answer. In our practice, if a local excision is not an immediate option at presentation, we start neoadjuvant imatinib. This allows us to minimize the need for more extensive resections. However, the local approach should factor in the specific pattern of growth and rectal wall involvement of these tumors. The additional choice between a transanal procedure, a perineal technique, and a more extensive approach has to be made carefully on an individualized basis. wedge resection of the lateral wall of the lower rectum via an in-line stapler. $v$ Final stapled suture. $v i$ Gross specimen

In conclusion, both top-level multidisciplinary expertise and top-level surgical expertise are needed if clinicians aim to improve the outcome for patients affected by anorcetal GISTs together with their quality of life.

\section{REFERENCES}

1. Hawkins AT, Wells KO, Krishnamurty DM, et al. Preoperative chemotherapy and survival for large anorectal GIST: a national analysis of 333 cases. Ann Surg Oncol. 2016. doi:10.1245/s10434016-5706-1.

2. Fiore M, Palassini E, Fumagalli E, et al. Preoperative imatinib mesylate for unresectable or locally advanced primary gastrointestinal stromal tumors (GIST). Eur $J$ Surg Oncol. 2009;35:739-45.

3. Dematteo RP, Ballman KV, Antonescu CR, et al. Adjuvant imatinib mesylate after resection of localised, primary gastrointestinal stromal tumour: a randomised, double-blind, placebocontrolled trial. Lancet. 2009;373:1097-104. 
4. Joensuu H, Eriksson M, Sundby Hall K, et al. Adjuvant imatinib for high-risk GI stromal tumor: analysis of a randomized trial. $J$ Clin Oncol. 2016;34:244-50.

5. Casali PG, Le Cesne A, Poveda Velasco A, et al. Time to definitive failure to the first tyrosine kinase inhibitor in localized GI stromal tumors treated with imatinib as an adjuvant: a European Organisation for Research and Treatment of Cancer Soft Tissue and Bone Sarcoma Group Intergroup Randomized Trial in collaboration with the Australasian Gastro-Intestinal Trials Group, UNICANCER, French Sarcoma Group, Italian Sarcoma Group, and Spanish Group for Research on Sarcomas. J Clin Oncol. 2015;33:4276-83.

6. Gronchi A, Raut CP. The combination of surgery and imatinib in GIST: a reality for localized tumors at high risk, an open issue for metastatic ones. Ann Surg Oncol. 2012;19:1051-5.

7. Jakob J, Mussi C, Ronellenfitsch U, Wardelmann E, Negri T, Gronchi A, Hohenberger P. Gastrointestinal stromal tumor of the rectum: results of surgical and multimodality therapy in the era of imatinib. Ann Surg Oncol. 2013;20:586-92. 